\title{
Frequency and Amplitude Identification of Weak Signal Based on the Limit System of Smooth and Discontinuous Oscillator
}

\author{
Haiping $\mathrm{Li}^{1,2} \cdot$ Ruilan Tian $^{1} \cdot$ Xiaolong Zhang ${ }^{1} \cdot$ Xinwei Yang $^{3}$
}

Received: 6 December 2021 / Accepted: 27 January 2022 / Published online: 21 February 2022

(c) The Author(s) 2022

\begin{abstract}
In this paper, a new method is proposed to identify the frequency and amplitude of weak signals by using a non-smooth system. The variable scale limit system of smooth and discontinuous (SD) oscillator without considering the phase is adopted as the identification system. By using the non-smooth stochastic subharmonic-like Melnikov method, an analytical expression of chaotic threshold under Gaussian white noise is given. Based on the phase diagram and Poincaré section diagram, the influence of noise intensity on the recognition system is studied. According to the non-smooth variable scale-convex-peak frequency identification method, the frequency of the signal to be detected can be accurately identified. Using the numerical simulation, the amplitude of the signal to be measured is identified according to the amplitude bifurcation diagram of the reference signal. The optimal value range of the parameters of the identification system is determined. Through an example of early fault of wheelset bearing of high-speed train, the frequency and amplitude of the early weak fault signal can be identified and the fault location can be determined, which verifies the effectiveness of the above method. The results show that the non-smooth system can identify the frequency and amplitude of the weak signal submerged in strong noise, and it has a wider application and higher accuracy than the continuous system.
\end{abstract}

Keywords Smooth and discontinuous oscillator · Frequency · Amplitude · Variable scale-convex-peak frequency identification method $\cdot$ Stochastic subharmonic-like Melnikov function

Ruilan Tian

tianrl@stdu.edu.cn

1 Department of Engineering Mechanics, Shijiazhuang Tiedao University, Shijiazhuang 050043, People's Republic of China

2 School of Science, Hebei University of Science and Technology, Shijiazhuang 050018, People's Republic of China

3 Department of Rail Transport, Shijiazhuang Institute of Railway Technology, Shijiazhuang 050041, People's Republic of China 


\section{Introduction}

Weak signal identification is widely used in many fields, such as universe exploration, fault diagnosis, medical diagnosis, life search, etc. How to identify it from strong noise has always been a research hotspot in the academic field. The characteristics of weak harmonic signals are amplitude, frequency and phase. There are many research results on the identification of these parameters. At present, weak signal identification systems adopt continuous systems, such as Duffing system [1-3], the variable scale Duffing system [4, 5], the Vanderpol-Duffing system [6], the dual coupling Duffing system [7], L-Y system [8-10], the time-delay Mathieu-Duffing system [11], Lorenz system [12], etc. But there are many shortcomings: (1) The calculation process of the Melnikov function of the continuous system is more complicated; (2) The calculation of the chaotic threshold often adopts necessary conditions, and the result is not accurate enough.

Moreover, many scholars have made a lot of achievements in the study of nonsmooth systems. Kunze et al. [13] introduced plane non-smooth systems comprehensively. Du et al. [14] proposed homoclinic bifurcation Melnikov function of nonsmooth systems and subharmonic Melnikov functions of non-smooth periodic orbits on the first and second kinds. Battelli and Feckan [15] gave Melnikov method for a class of high-dimensional discontinuous systems. Granados et al. [16] considered a two-dimensional piecewise smooth system divided into two regions by a transformation manifold. Granados et al. [17] studied the scattering maps of two coupled piecewise smooth systems, and their numerical applications on rocking blocks and Melnikov method. Tian et al. [18] used the pendulum as a model to study the chaotic thresholds under different pulse excitations when the pendulum collided with a rigid wall by using the non-smooth Melnikov method. Li et al. [19] studied a twodimensional piecewise smooth system divided into four regions by two conversion surfaces. At present, there is no paper studying weak signal identification based on non-smooth system. Hence, it is the main task of this paper to find a non-smooth system with simple Melnikov function and accurate expression of chaotic threshold.

Consider the limit system of smooth and discontinuous (SD) oscillator which first proposed by Cao et al. [20]

$$
\ddot{x}+[x-\operatorname{sign}(x)]=0 .
$$

Many scholars have studied system (1) [21-25]. It can be seen that the limit system of the SD oscillator has an abundant of dynamic behavior transitions. From its simple non-smooth equation form, the calculation process of Melnikov will be simpler. So, we will consider the limit system of the SD oscillator as the identification system.

In this paper, when the variable scale limit system of SD oscillator without considering the phase is used as the identification system to identify weak signal parameters, the paper is organized as follows. In the next section, the limit system of SD oscillator with Gaussian white noise is adopted as the basic identification system. By using the non-smooth stochastic subharmonic-like Melnikov method, according to the necessary and sufficient condition that Melnikov function has a simple 
zero point in the mean square sense, the analytical expression of chaotic threshold of the identification system is given. The influence of noise intensity on the identification system is studied by making phase diagrams and Poincaré section diagrams. In Sect. 3, the expression of chaotic threshold of the identification system with signals to be detected is determined. Non-smooth variable-scale-convex-peak frequency identification method is used to identify the frequency of the signal to be detected. The amplitude of the signal to be detected is identify by using the bifurcation diagram of the amplitude of the reference signal. Determine the optimal value range of the parameter of the identification system. In Sect. 4, the electronic circuit is designed, which verifies the feasibility of the above method. In Sect. 5, the effectiveness of the above method is verified by an example of early fault of wheelset bearing in high-speed train.

\section{Parameter Identification Model}

Consider the limit system of SD oscillator with Gaussian white noise

$$
\left\{\begin{array}{l}
\dot{x}=y, \\
\dot{y}=-x+\operatorname{sign}(x)+\varepsilon(-2 \mu y+f \cos (\omega t)+\sigma n(t)),(x, y) \neq(0,0),
\end{array}\right.
$$

where $-x+\operatorname{sign}(x)$ is the nonlinear term, $\mu$ is the damping coefficient, $f \cos (\omega t)$ is the reference signal, $\sigma n(t)$ is Gaussian white noise with intensity $\sigma$ and power spectral density $S_{n}(\omega)=K=\frac{\sigma^{2}}{2 \pi}$.

The undisturbed system of system (2) is

$$
\left\{\begin{array}{l}
\dot{x}=y \\
\dot{y}=-x+\operatorname{sign}(x),(x, y) \neq(0,0) .
\end{array}\right.
$$

System (3) has three singularities $(0,0),( \pm 1,0)$. Because system (3) is discontinuous at $(0,0)$, and the Jacobian matrix does not exist, the topological structure near the saddle-like point $(0,0)$ is similar to the saddle point. The Jacobian matrix is $J_{( \pm 1,0)}=\left(\begin{array}{cc}0 & 1 \\ -1 & 0\end{array}\right)$ at $( \pm 1,0)$, and its eigenvalue is $\lambda_{1,2}= \pm i$. So $( \pm 1,0)$ is the center.

The piecewise Hamilton function of system (3) is

$$
H(x, y)=\frac{y^{2}}{2}+U=\frac{y^{2}}{2}+\frac{x^{2}}{2}-|x|
$$

where $U=\frac{x^{2}}{2}-|x|$ is the potential energy function, as shown in Fig. 1 .

When $H(x, y)=0$, the homoclinic-like orbits of system (3) yield (5), as shown in Fig. 2.

$$
q_{ \pm}=\left(x_{ \pm}(t), y_{ \pm}(t)\right)=( \pm \cos t \pm 1, \mp \sin t), t \in[-\pi, \pi] .
$$

The stochastic subharmonic-like Melnikov function of system (2) is 
Fig. 1 The potential energy function of Eq. (4)
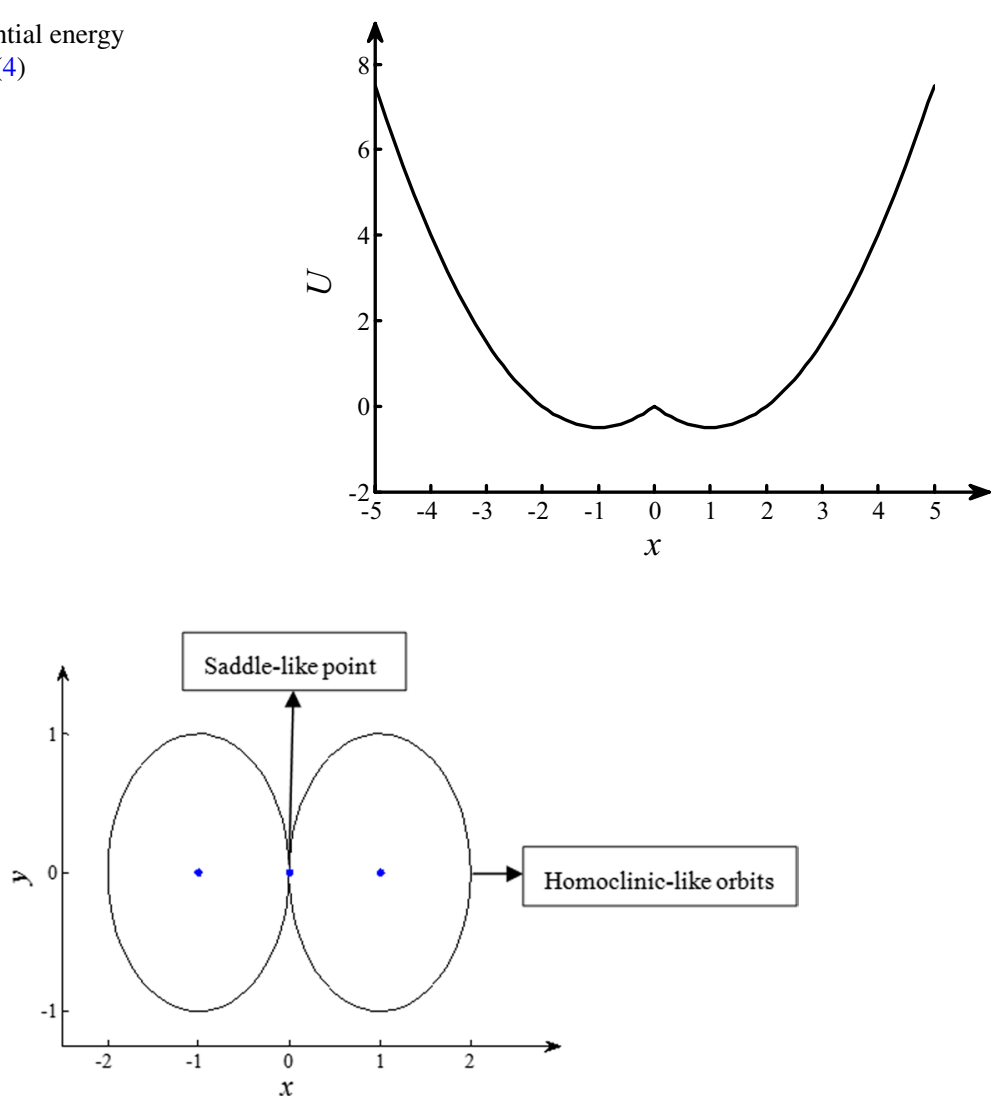

Fig. 2 Saddle-like point and homoclinic-like orbits

$$
M_{+}\left(t_{0}\right)=\int_{-\pi}^{\pi} y_{+}(t)\left[-2 \mu y_{+}(t)+f \cos \left(\omega\left(t+t_{0}\right)\right)+\sigma n\left(t+t_{0}\right)\right] d t=-M+\bar{M}\left(t_{0}\right)+\tilde{M}\left(t_{0}\right),
$$

where $M=2 \mu \int_{-\pi}^{\pi} y_{+}^{2}(t) d t=2 \mu \pi, \bar{M}\left(t_{0}\right)=f \int_{-\pi}^{\pi} y_{+}(t) \cos \left[\omega\left(t+t_{0}\right)\right] d t=\frac{2}{1-\omega^{2}} f \sin (\omega \pi) \sin \left(\omega t_{0}\right)$. $\tilde{M}\left(t_{0}\right)$ is the random part of the stochastic subharmonic-like Melnikov function. The characteristics of $\tilde{M}\left(t_{0}\right)$ considered below. Since $n\left(t+t_{0}\right)$ is a stationary random process as the input, the output of $\tilde{M}\left(t_{0}\right)$ is also a stationary random process. Therefore, the statistical characteristics of the stochastic Melnikov function can be completely determined by knowing the first and the second moment.

The first moment of $\tilde{M}\left(t_{0}\right)$ is

$$
E\left[\tilde{M}\left(t_{0}\right)\right]=E\left[\int_{-\pi}^{\pi} \sigma y_{+}(t) n\left(t+t_{0}\right) d t\right]=\int_{-\pi}^{\pi} \sigma y_{+}(t) E\left[n\left(t+t_{0}\right)\right] d t=0 .
$$

Since $E\left[\tilde{M}\left(t_{0}\right)\right]=0$, the first moment ignores the influence of noise on the chaos threshold. Hence, the second moment of $M_{+}\left(t_{0}\right)$ must be considered.

The second moment of $\tilde{M}\left(t_{0}\right)$ is 


$$
E\left[\tilde{M}^{2}\left(t_{0}\right)\right]=\int_{-\pi}^{\pi}|H(\omega)|^{2} S_{n}(\omega) d \omega
$$

where $H(\omega)$ is the transfer function. Impulse response function $h(t)=\sigma y_{+}(t)$ and $H(\omega)$ form a Fourier transform pair, and we have

$$
H(\omega)=\int_{-\pi}^{\pi} h(t) e^{-i \omega t} d t=-\sigma \int_{-\pi}^{\pi} \sin (t) e^{-i \omega t} d t=\frac{2 i \sigma}{1-\omega^{2}} \sin (\omega \pi),
$$

then

$$
E\left[\tilde{M}^{2}\left(t_{0}\right)\right]=\int_{-\pi}^{\pi}|H(\omega)|^{2} S_{n}(\omega) d \omega=8 K \sigma^{2} \int_{0}^{\pi} \frac{\sin ^{2}(\omega \pi)}{\left(1-\omega^{2}\right)^{2}} d \omega=8 K \sigma^{2} A,
$$

where $A=\int_{0}^{\pi} \frac{\sin ^{2}(\omega \pi)}{\left(1-\omega^{2}\right)^{2}} d \omega$. In the mean square sense, the necessary and sufficient conditions for the stochastic subharmonic-like Melnikov function of system (2) to have a simple zero point are

$$
\left\{\begin{array}{c}
E\left[M_{+}^{2}\left(t_{0}\right)\right]=-(2 \mu \pi)^{2}+\left(\frac{2}{1-\omega^{2}} f \sin (\omega \pi) \sin \left(\omega t_{0}\right)\right)^{2}+8 K \sigma^{2} A=0, \\
\frac{d E\left[M_{+}^{2}\left(t_{0}\right)\right]}{d t_{0}}=\frac{8 \omega}{\left(1-\omega^{2}\right)^{2}} f^{2} \sin ^{2}(\omega \pi) \sin \left(\omega t_{0}\right) \cos \left(\omega t_{0}\right) \neq 0 .
\end{array}\right.
$$

The chaotic threshold of system (2) is

$$
f_{d}=\frac{\left(1-\omega^{2}\right) \sqrt{(\mu \pi)^{2}-2 K \sigma^{2} A}}{\sin (\omega \pi) \sin \left(\omega t_{0}\right)},
$$

satisfied $\omega \neq k, t_{0} \neq k \pi / \omega, t_{0} \neq(2 k \pi+\pi / 2) / \omega, k=0, \pm 1, \pm 2, \ldots, \pm n, \ldots$

Let $\mu=0.1, t_{0}=2$. Accroding to Eq. (12), make the function diagram of the chaotic threshold $f_{d}$ with respect to reference signal frequency $\omega$ and noise intensity $\sigma$ as shown in Fig. 3.

If $f>f_{d}, \varepsilon>0$ and it is sufficiently small, system (2) may appear chaos. From the point of view of the calculation process, the calculation of the Melnikov function is simpler than that of the Duffing system. When solving the chaos threshold,

Fig. 3 The function diagram of chaotic threshold $f_{d}$ with respect to reference signal frequency $\omega$ and noise intensity $\sigma$

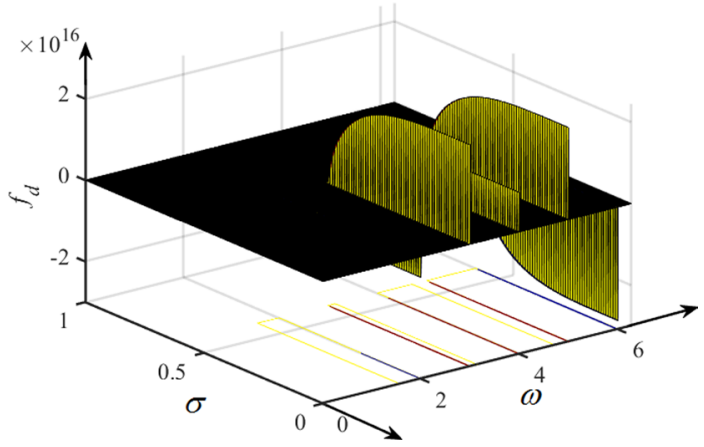


the necessary and sufficient conditions are used, which are more accurate than the necessary conditions.

It can be seen from Eq. (12) that noise has an impact on the threshold which is verified by numerical simulation. Let $\mu=0.1, f=0.6, \omega=1.2$ make the phase diagram and Poincaré section diagram of system (2) with different noise intensity $\sigma$, as shown in Fig. 4.

It can be seen from Fig. 4a that system (2) have periodic five motion when $\sigma=0$, and the phenomenon of dissipation will appear with the increase of the noise intensity. Figure $4 \mathrm{~b}$, c show the multiply periodic motion when $\sigma=0.01$ and $\sigma=0.05$. Figure $4 \mathrm{~d}$ becomes chaotic motion when $\sigma=0.5$. Figure $4 \mathrm{e}$ shows the Poincaré section diagram of system (2) when $\sigma=0.5$.

\section{Parameter Identification of Weak Signal}

\subsection{Parameter Identification System}

The signal to be detected without considering the phase is added into system (2) to obtain

$$
\left\{\begin{array}{l}
\dot{x}=y, \\
\dot{y}=-x+\operatorname{sign}(x)+\varepsilon\left(-2 \mu y+f \cos (\omega t)+r \cos \left(\omega_{1} t\right)+\sigma n(t)\right),(x, y) \neq(0,0),
\end{array}\right.
$$

where $r \cos \left(\omega_{1} t\right)$ is the signal to be detected.

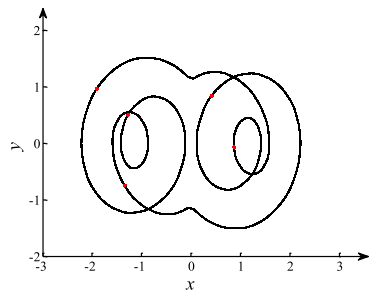

(a) $\sigma=0$

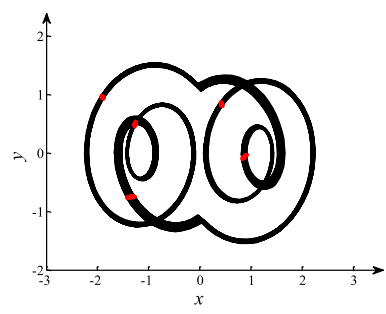

(b) $\sigma=0.01$

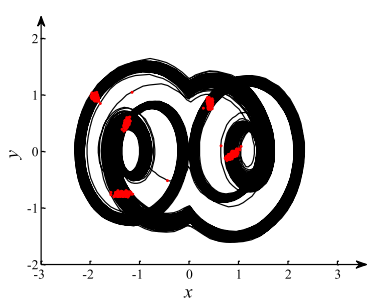

(c) $\sigma=0.05$

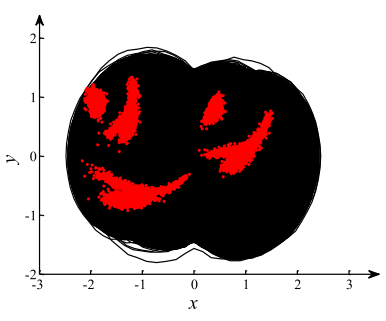

(d) $\sigma=0.5$

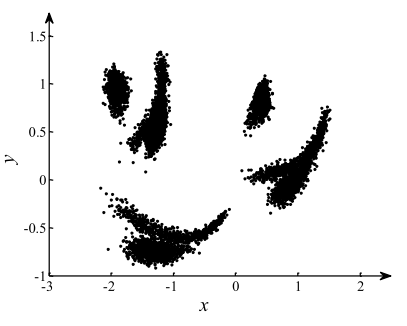

(e) $\sigma=0.5$

Fig. 4 Phase diagram and Poincaré section diagram of system (2) with different noise intensity 
Using the same method as in Ref. [5], making scale transformation on system (13), we get the following identification system

$$
\left\{\begin{array}{l}
\dot{x}=\omega y, \\
\dot{y}=\omega\left(-x+\operatorname{sign}(x)+\varepsilon\left(-2 \mu y+f \cos (\omega t)+r \cos \left(\omega_{1} t\right)+\sigma n(t)\right)\right),(x, y) \neq(0,0) .
\end{array}\right.
$$

When $\omega=\omega_{1}$, the chaotic threshold of systems (13) and (14) are same and yields

$$
f_{d}^{\prime}=\frac{\left(1-\omega^{2}\right) \sqrt{(\mu \pi)^{2}-2 K \sigma^{2} A}}{\sin (\omega \pi) \sin \left(\omega t_{0}\right)}-r
$$

where $\omega \neq k, t_{0} \neq k \pi / \omega, t_{0} \neq(2 k \pi+\pi / 2) / \omega, k=0, \pm 1, \pm 2, \ldots, \pm n, \ldots$.

So, the amplitude of the weak signal to be detected is

$$
r=f_{d}^{\prime}-f_{d}
$$

When $\omega \neq \omega_{1}$, set $\omega_{1}=\omega+\Delta \omega$, consider the chaotic threshold of system (13), we have

$$
\begin{aligned}
E\left[M_{+}^{2}\left(t_{0}\right)\right]=-M^{2}+\bar{M}^{2}\left(t_{0}\right)+E\left[\tilde{M}^{2}\left(t_{0}\right)\right] \\
=-(2 \mu \pi)^{2}+2\left\{\frac{f}{1-\omega^{2}} \sin (\omega \pi) \sin \left(\omega t_{0}\right)+\frac{r}{1-\omega_{1}^{2}} \sin \left(\omega_{1} \pi\right) \sin \left[(\omega+\Delta \omega) t_{0}\right]\right\}^{2}+8 K \sigma^{2} A \\
=-(2 \mu \pi)^{2}+8 K \sigma^{2} A+2\left\{\left[\frac{f}{1-\omega^{2}} \sin (\omega \pi)+\frac{r}{1-\omega_{1}^{2}} \sin \left(\omega_{1} \pi\right) \cos \left(\Delta \omega t_{0}\right)\right] \sin \left(\omega t_{0}\right)\right. \\
\left.\left.+\frac{r}{1-\omega_{1}^{2}} \sin \left(\omega_{1} \pi\right) \sin \left(\Delta \omega t_{0}\right) \cos \left(\omega t_{0}\right)\right)\right\} \\
=-(2 \mu \pi)^{2}+8 K \sigma^{2} A+2 F \sin \left(\omega t_{0}+\theta\right),
\end{aligned}
$$

where

$$
\begin{aligned}
& F=\sqrt{\left(\frac{f}{1-\omega^{2}} \sin (\omega \pi)+\frac{r}{1-\omega_{1}^{2}} \sin \left(\omega_{1} \pi\right) \cos \left(\Delta \omega t_{0}\right)\right)^{2}+\left(\frac{r}{1-\omega_{1}^{2}} \sin \left(\omega_{1} \pi\right) \sin \left(\Delta \omega t_{0}\right)\right)^{2}}, \\
& \theta=\tan \frac{\frac{r}{1-\omega_{1}^{2}} \sin \left(\omega_{1} \pi\right) \sin \left(\Delta \omega t_{0}\right)}{\frac{f}{1-\omega^{2}} \sin (\omega \pi)+\frac{r}{1-\omega_{1}^{2}} \sin \left(\omega_{1} \pi\right) \cos \left(\Delta \omega t_{0}\right)} .
\end{aligned}
$$

The chaotic threshold of system (13) is

$$
f_{d}^{\prime}=\frac{\left(1-\omega^{2}\right)}{\sin (\omega \pi)}\left(\frac{1}{\sin \left(\omega t_{0}+\theta\right)} \sqrt{(\mu \pi)^{2}-2 K \sigma^{2} A}-\frac{r}{1-\omega_{1}^{2}} \sin \left(\omega_{1} \pi\right)\right),
$$

satisfied $\omega \neq k, t_{0} \neq k \pi / \omega, t_{0} \neq(2 k \pi+\pi / 2) / \omega, k=0, \pm 1, \pm 2 \cdots, \pm n, \cdots$.

The chaotic threshold of systems (14) is the same as that of (13), and the roof is similar to that of (13), which is omitted here.

Let $\mu=0.1, f=0.6, r=0.02, \omega_{1}=0.7$, the bifurcation diagrams of systems (13) and (14) of the reference signal frequency $\omega$ are shown in Fig. 5. 


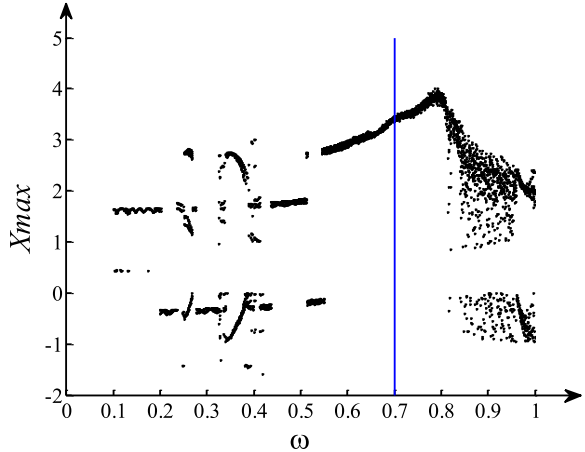

(a) system (13)

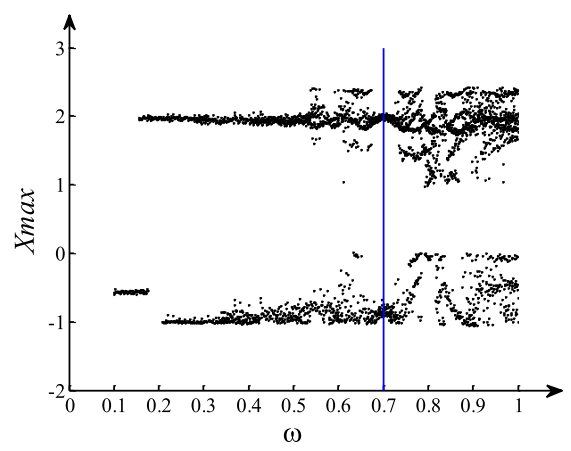

(b) system (14)

Fig. 5 The bifurcation diagram of different systems of reference signal frequency $\omega$

Fig. 6 Bifurcation diagram of system (14) of reference signal frequency $\omega$

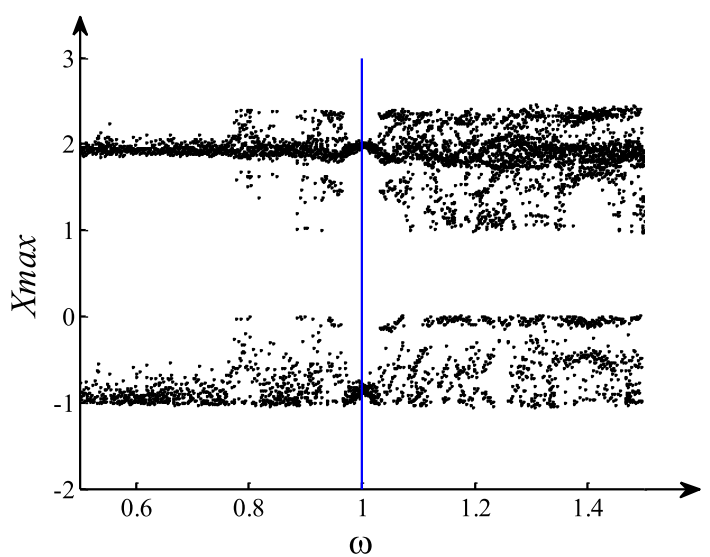

From Fig. 5, it can be seen that there is not a convex peak at $\omega=0.7$ of system (13), while there is an obvious convex peak at $\omega=0.7$ of the variable scale system (14). So, the variable scale system can be used to identify weak signals. This paper uses the variable scale system (14) to identify weak signal frequency and amplitude.

\subsection{Frequency Identification of Weak Signal}

Let $\mu=0.1, f=0.6, r=0.02, \omega_{1}=1$, the bifurcation diagram of system (14) of reference signal frequency $\omega$ is shown in Fig. 6.

As can be seen from Fig. 6 , there is a convex peak phenomenon at $\omega=1$, which shows that the frequency of the signal to be detected is $\omega_{1}=1$. The method of identifying frequencies based on a non-smooth variable-scale system by making a frequency 
bifurcation diagram of the signal to be detected is called a non-smooth variable-scale convex peak frequency identification method.

\subsection{Amplitude Identification of Weak Signal}

Let $\mu=0.05, r=0.02, \omega=\omega_{1}=1.06066$ bifurcation diagrams of the reference signal amplitude $f$ of systems (2) and (14) are shown in Fig. 7.

It can be seen from Fig. 7a that the chaotic threshold of system (2) is $f_{d}=0.1$ (shown by the blue line), which is obviously different from $f_{d}^{\prime}=0.0805$ of system (14) in Fig. $7 b$ (shown by the red line). So, the amplitude of the weak signal to be detected is $r^{\prime}=f_{d}-f_{d}^{\prime}=0.0195$, which is same as hypothesis $r=0.02$. The absolute error is 0.0005 and the relative error is $2.5 \%$.

The phase diagram of system (2) changing with the reference signal amplitude $f$ are shown in Fig. 8a-c. The phase diagram of system (14) changing with the reference signal amplitude $f$ are shown in Fig. 8d-f.

Figure $8 \mathrm{a}-\mathrm{c}$ show that system (2) changes from periodic motion to chaotic motion, and the chaotic threshold is $f_{d}=0.1$. Figure $8 \mathrm{~d}-\mathrm{f}$ show that system (14) changes from periodic motion to the chaotic motion, and the chaotic threshold is $f_{d}^{\prime}=0.08$. So, the amplitude of the signal to be detected is $r^{\prime}=f_{d}-f_{d}^{\prime}=0.02$, which verifies the correctness of the bifurcation diagram to identify the amplitude. After verification by numerical simulation, the optimal value range of system (14) is shown in Table 1.

From Table 1 we can see, the phenomenon of convex peak appears when $r_{\min }=0.02$ and $\sigma_{\max }=0.18$, so the signal-to-noise ratio of system (14) is

$$
S / N=10 \lg \frac{r_{\min }^{2}}{2 \sigma_{\max }^{2}}=-50.8757 \mathrm{~dB} .
$$

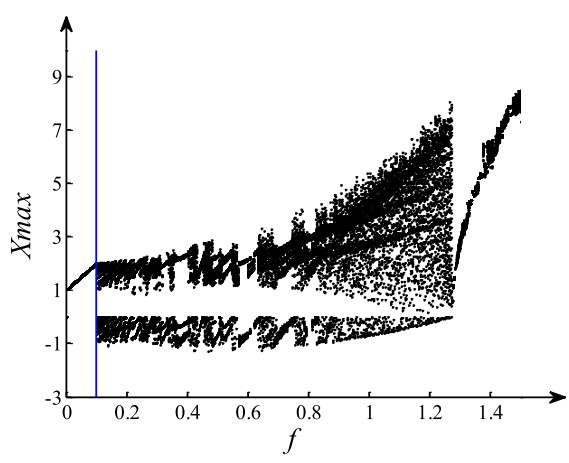

(a) system (2)

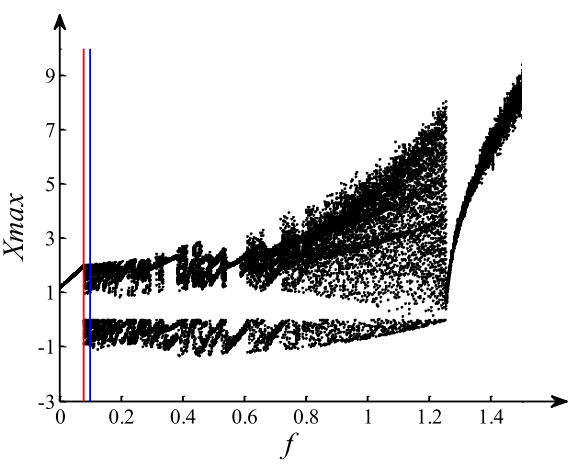

(b) system (14)

Fig. 7 Bifurcation diagram of different system of the reference signal amplitude $f$ 


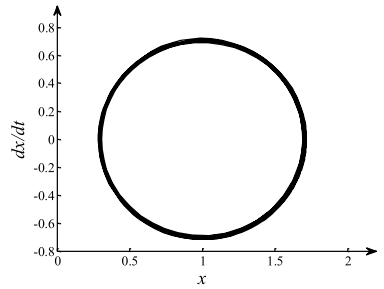

(a) $f=0.09$

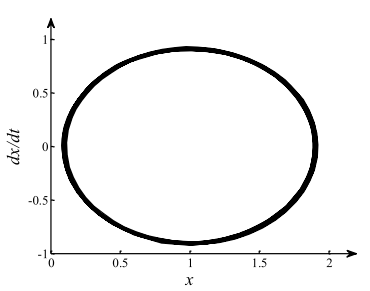

(d) $f=0.07$

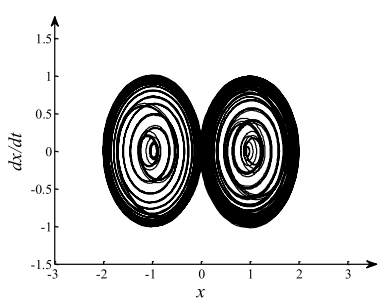

(b) $f=0.1$

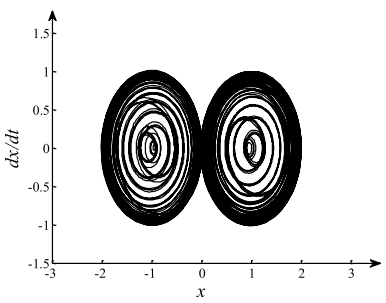

(e) $f=0.08$

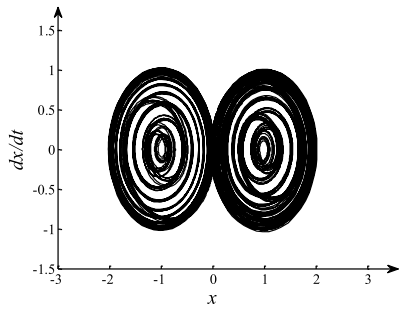

(c) $f=0.11$

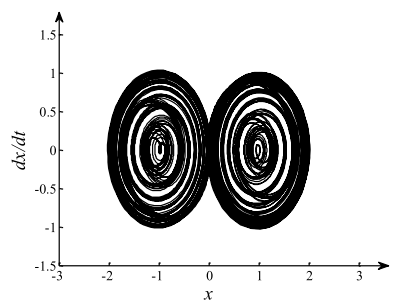

(f) $f=0.09$

Fig. 8 a-c Phase diagrams of system (2) about different amplitudes $f$; $\mathbf{d}-\mathbf{f}$ phase diagram of the system (14) about different amplitudes $f$

\section{Circuit Design and Simulation}

The nonlinear analog circuit is constructed by Multisim software. Operational amplifier TL082 and analog multiplier AD633JN are shown in Fig. 9. Module V1 represents the reference signal, module $\mathrm{V} 2$ represents the signal to be detected and module V3 represents Gaussian white noise. Operational amplifier $\mathrm{U} 2 \mathrm{~A}$ and resistor $R_{1}, R_{2}$ constitute a zero-crossing comparator, which together with resistor $R_{3}, R_{4}$ and operational amplifier U2B are equivalent to a sign function.

A bifurcation diagram about the frequency and amplitude of the reference signal is made by using Fig. 9 to verify the feasibility of identifying the frequency and amplitude of the signal to be detected by using this bifurcation diagram. According to the theoretical results, suppose the damping coefficient $\mu=0.1$, the amplitude of the signal to be detected $r=0.05$, the noise intensity $\sigma=0.01$, the reference signal amplitude $f=0.6$, the frequency to be detected $\omega_{1}=1.2$, the bifurcation diagram of the frequency $\omega$ of

Table 1 The optimal value range of the parameter of the identification system (14)

\begin{tabular}{llllll}
\hline Parameter & $\begin{array}{l}\text { The amplitude } \\
\text { of the reference } \\
\text { signal } f\end{array}$ & $\begin{array}{l}\text { The damping } \\
\text { coefficient } \mu\end{array}$ & $\begin{array}{l}\text { The amplitude } \\
\text { reference signal } r\end{array}$ & $\begin{array}{l}\text { Reference signal } \\
\text { frequency } \omega_{1}\end{array}$ & $\begin{array}{l}\text { Intensity of } \\
\text { noise } \sigma\end{array}$ \\
\hline $\begin{array}{l}\text { Value } \\
\text { range }\end{array}$ & {$[0.55,0.75]$} & {$[0.05,5]$} & {$[0.02,10]$} & {$[0,+\infty)$} & {$[0,0.18]$} \\
\hline
\end{tabular}


the signal to be detected of system (14) is shown in Fig. 10a. Suppose the reference signal frequency $\omega=1.2$, the bifurcation diagram of the reference signal amplitude $f$ of system (14) is shown in Fig. 10b. As can be seen from Fig. 10, Fig. 9 can be used to identify the frequency and amplitude of weak signals.

\section{Experimental Verification of Parameter Identification for Early Fault Signal of Wheelset Bearing in High-speed Train}

The experimental data come from Shijiazhuang Tiedao University State Key Laboratory of Mechanical Behavior and System Safety of Traffic Engineering Structures. The bearing type is FAG F-80781109 Tarol 130/240-b-TVP. The outer diameter, inner diameter, roller diameter, roller number and pressure angle are $240 \mathrm{~mm}$, $130 \mathrm{~mm}, 26.5 \mathrm{~mm}, 17,10^{\circ}$ respectively. The outer ring of the bearing has a machining failure with length $5 \mathrm{~mm}$, width $1 \mathrm{~mm}$, high $0.7 \mathrm{~mm}$. The speed of bearing is $1200 \mathrm{r} / \mathrm{min}$. This experiment contains $1.5 \times 10^{5}$ data. The weak fault signal is submerged in strong noise completely in the waveform diagram (Fig. 11).

The theoretical failure frequency calculated according to bearing parameters and rotational speed are shown in Table 2.

\subsection{Experimental Verification of Frequency Identification for Early Fault Signal}

Input the fault experimental data of the bearing into system (14) to make the bifurcation diagram of the reference signal frequency $\omega$ of the signal to be detected, as shown in Fig. 12.

\subsection{Experimental Verification of Amplitude Identification for Early Fault Signal}

Input the fault-free experimental data of the bearing into system (2) to make the bifurcation diagram of the reference signal amplitude $f$, as shown in Fig. 13a. Input the fault experimental data of the bearing into system (14) to make the bifurcation diagram of the reference signal amplitude $f$, as shown in Fig. $13 \mathrm{~b}$.

It can be seen from Fig. 13a that the amplitude of the reference signal is $f_{e}=0.1778$ when there is no fault in the bearing; it can be obtained from Fig. $13 \mathrm{~b}$ that the amplitude of the reference signal is $f_{e}^{\prime}=0.1651$ when there is a fault in the bearing. Then, the amplitude of the signal to be detected is $r_{e}=f_{e}-f_{e}^{\prime}=0.0127$.

\section{Conclusion}

In this paper, the limit system of SD oscillator without considering the phase is selected as the identification system. According to the random subharmonic-like Melnikov method, the analytical expression of the chaotic threshold is given. The 


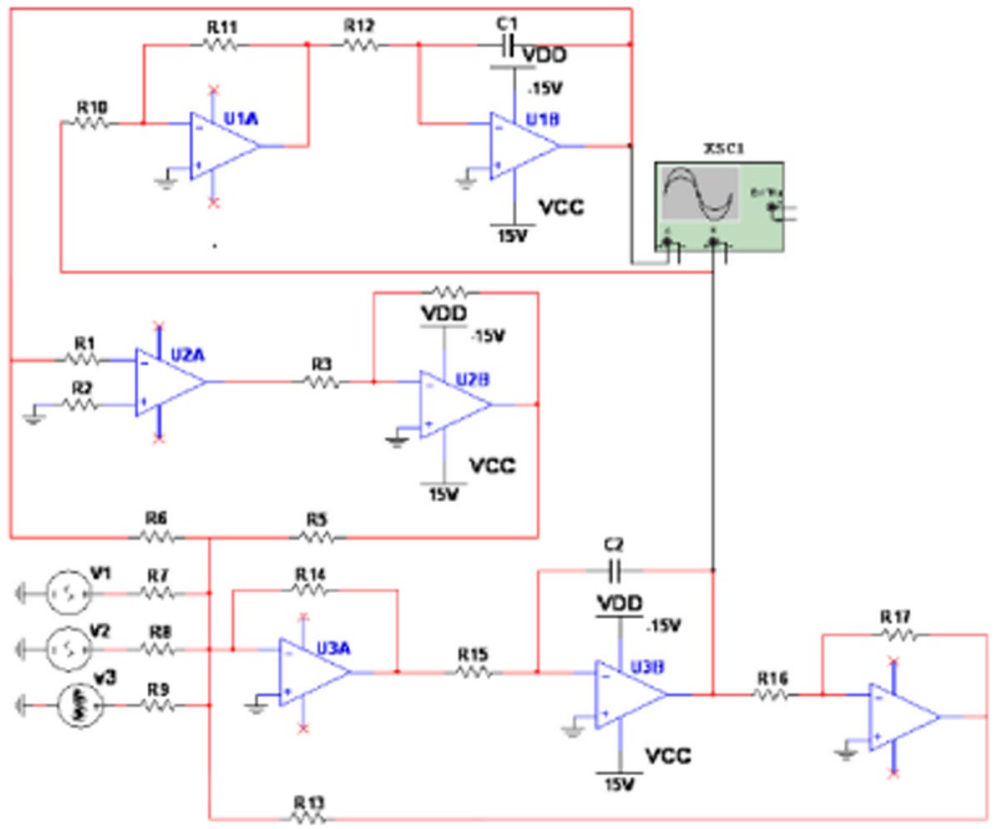

Fig. 9 Analog circuit diagram of system (14)

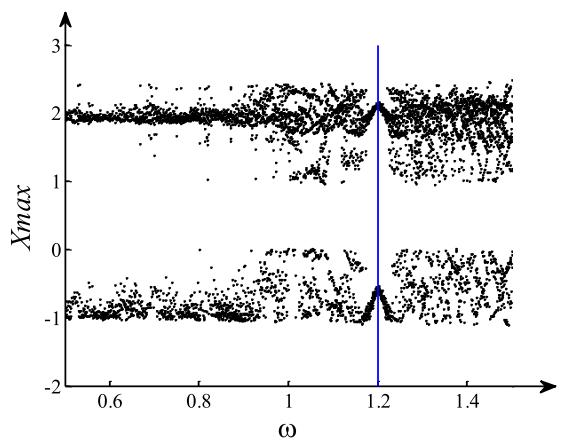

(a)

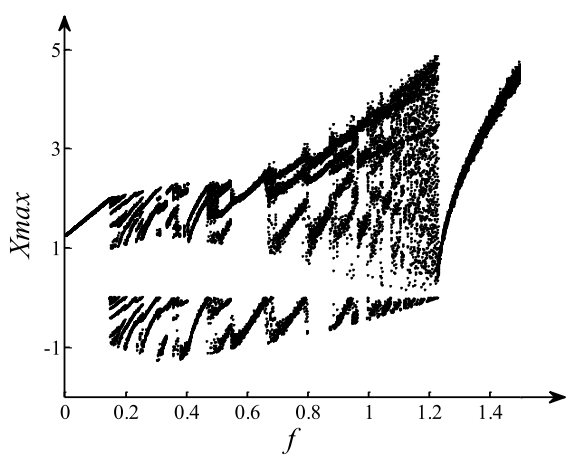

(b)

Fig. 10 a Bifurcation diagram of system (14) of the frequency $\omega$ of the signal to be detected; $\mathbf{b}$ bifurcation diagram of system (14) of the reference signal amplitude $f$

calculation process of random subharmonic Melnikov function of non-smooth system is simpler and more concise than that of continuous system, which is more suitable for parameter identification of weak signal. More importantly, using the sufficient and necessary conditions for the existence of simple zeros of the random subharmonic-like Melnikov function of the non-smooth system, a more accurate 


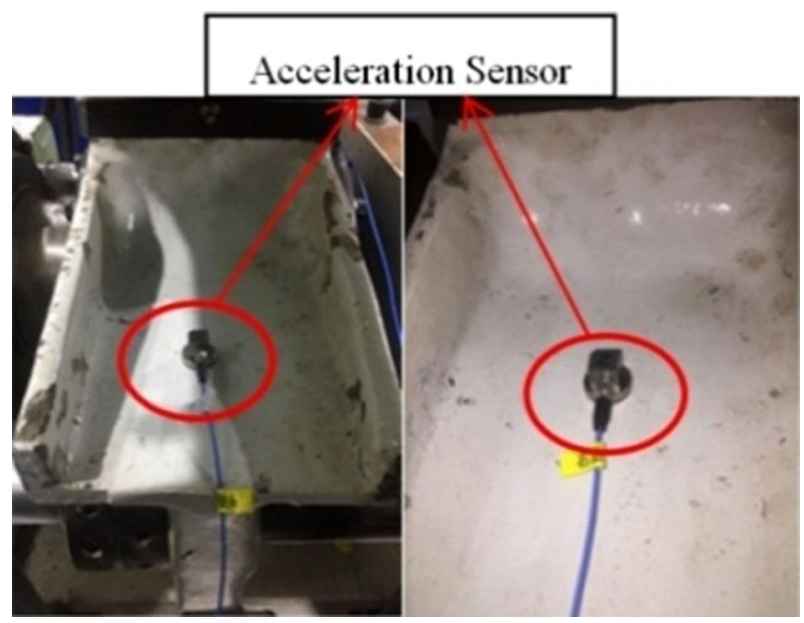

Fig. 11 Layout of measuring points of wheelset bearings

Table 2 Theoretical calculation of fault frequency

\begin{tabular}{lllll}
\hline Fault location & Outer ring & Inner ring & Rolling element & Cage \\
\hline Frequency $(\mathrm{Hz})$ & 146.03 & 193.97 & 68.42 & 8.59 \\
Angular frequency $(\mathrm{rad} / \mathrm{s})$ & 917 & 1218 & 430 & 54 \\
\hline
\end{tabular}

chaotic threshold is obtained, which lays a theoretical foundation for the identification of frequency and amplitude. Furthermore, it is clarified that the limit system of the variable-scale SD oscillator can be used as a frequency identification system. The non-smooth variable scale convex peak frequency identification method is used to identify the frequency of the signal to be detected, and the signal-to-noise ratio is $-50.8757 \mathrm{~dB}$. Through numerical simulation, the results obtained by identifying the amplitude of the signal to be measured using the amplitude bifurcation diagram of the reference signal are the same as those obtained by determining the amplitude through the phase diagram. The absolute error is 0.0195 and the relative error is $2.5 \%$. The electronic circuit is designed to verify the feasibility of the bifurcation diagram to identify the amplitude and the convex peak method to identify the frequency. The optimal value range of the parameters of the identification system is determined. Taking an early bearing fault of a high-speed train wheel set as an example. In frequency identification, it is judged that the bearing outer ring may fail by observing the center value of the convex peak with the absolute error of $31.5 \mathrm{rad} / \mathrm{s}$ and the relative error of $3.44 \%$, which fully verifies the effectiveness of the above method. In amplitude bifurcation, the amplitude of the weak signal can be accurately identified by the amplitude difference in the amplitude bifurcation diagram of the reference signal. The results show that the non-smooth system can identify the frequency and amplitude of the weak signal submerged in strong noise. The Melnikov function is simple to be calculated. The non-smooth variable 
Fig. 12 Bifurcation diagram of system (14) about the frequency $\omega$ of the signal to be detected

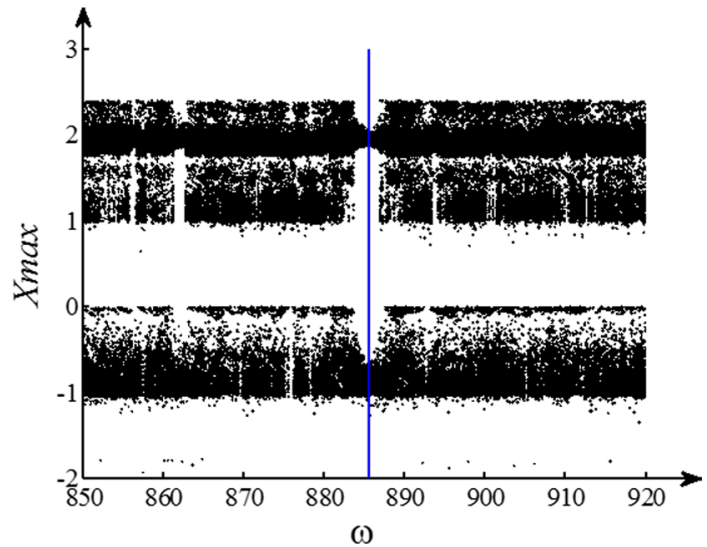

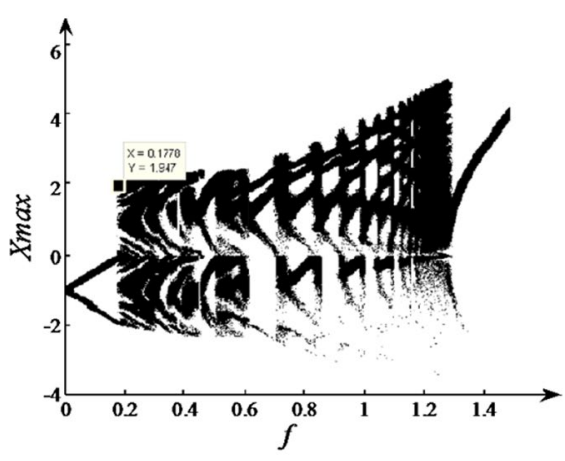

(a) $\operatorname{system}(2)$

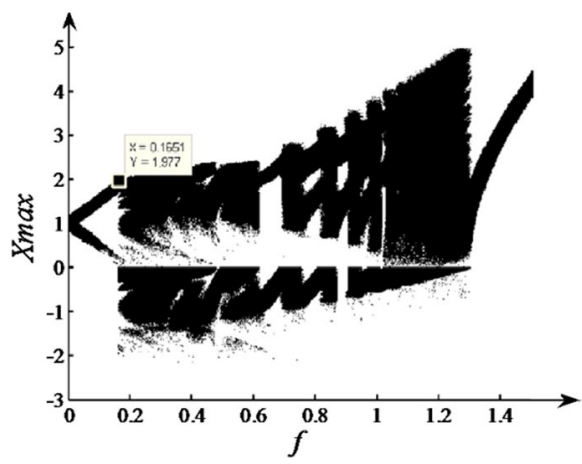

(b) $\operatorname{system~(14)~}$

Fig. 13 Bifurcation diagram of different system of the reference signal amplitude $f$

scale-convex-peak frequency identification method has higher accuracy and is more widely used than continuous systems.

Author Contributions HL study conceptualization and writing the manuscript. RT supervision. XZ circuit design. XY software. All authors read and approved the final manuscript.

Funding This work is financially supported by the National Natural Science Foundation of China (Nos. 11872253, 12072203, 12102274, 11602151), "333 Talents Project" of Hebei Province (No. A202005007), the Hundred Excellent Innovative (No. SLRC2019037).

Data Availability The authors confirm that the data supporting the findings of this study are available within the article and its supplementary materials.

\section{Declarations}

Conflict of interest The authors declare that they have no conflict of interest. 
Open Access This article is licensed under a Creative Commons Attribution 4.0 International License, which permits use, sharing, adaptation, distribution and reproduction in any medium or format, as long as you give appropriate credit to the original author(s) and the source, provide a link to the Creative Commons licence, and indicate if changes were made. The images or other third party material in this article are included in the article's Creative Commons licence, unless indicated otherwise in a credit line to the material. If material is not included in the article's Creative Commons licence and your intended use is not permitted by statutory regulation or exceeds the permitted use, you will need to obtain permission directly from the copyright holder. To view a copy of this licence, visit http://creativecommons.org/licen ses/by/4.0/.

\section{References}

1. Zhai, D.Q., Liu, C.X., Liu, Y., Xu, Z.: Determination of the parameters of unknown signals based on intermittent chaos. Acta Phys. Sin. 59(2), 816-825 (2010)

2. Niu, D.Z., Chen, C.X., Ban, F., Xu, H.X., et al.: Blind angle elimination method in weak signal detection with Duffing oscillator and construction of detection statistics. Acta Phys. Sin. 64(6), 060503 (2015)

3. Cong, C., Li, X.K., Song, Y.: A method of detecting line spectrum of ship-radiated noise using a new intermittent chaotic oscillator. Acta Phys. Sin. 63(6), 168-179 (2014)

4. Lai, Z.H., Leng, Y.G., Sun, J.Q., Fan, S.B.: Weak characteristic signal detection based on scale transformation of Duffing oscillator. Acta Phys. Sin. 59(2), 816-825 (2010)

5. Tian, R.L., Zhao, Z.J., Xu, Y.: Variable scale-convex-peak method for weak signal detection. Sci. China Technol. Sci. 64(2), 331-340 (2020)

6. Zhao, Z.H., Yang, S.P.: Application of Van der pol-Duffing oscillator in weak signal detection. Comput. Electr. Eng. 41, 1-8 (2015)

7. Wang, H.W., Cong, C.: A new signal detection and estimation method by using Duffing system. Acta Electron. Sin. 44(6), 1450-1457 (2016)

8. Li, Y., Yang, B.J.: Chaotic system for the detection of periodic signals under the background of strong noise. Chin Sci. Bull. 48, 508-510 (2003)

9. Liu, H.B., Wu, D.W., Dai, C.J., Mao, H.: A new weak sinusoidal signal detection method based on Duffing oscillators. Acta Phys. Sin. 41(1), 8-12 (2013)

10. Liu, H.B., Wu, D.W., Jin, W., Wang, Y.Q.: Study on weak signal detection method with Duffing oscillators. Acta Phys. Sin. 62(05), 34-39 (2013)

11. Wang, Q.B., Yang, Y.J., Zhang, X.: Weak signal detection based on Mathieu-Duffing oscillator with time-delay feedback and multiplicative noise. Chaos Soliton Fract 137, 109832 (2020)

12. Gokyildirim, A., Uyaroglu, Y., Pehlivan, I.: A week signal detection application based on hyperchaotic Lorenz system. Teh Vjesn 25(3), 701-708 (2018)

13. Kunze, M.: Non-smooth Dynamical Systems. Springer, Berlin (2000)

14. Du, Z.D., Zhang, W.N.: Melnikov method for homoclinic bifurcation in nonlinear impact oscillators. Comput. Math. Appl. 50(3-4), 445-458 (2005)

15. Battelli, F., Fečkan, M.: Homoclinic trajectories in discontinuous systems. J. Dyn. Differ. Equ. 20(2), 337-376 (2008)

16. Granados, A., Hogan, S.J., Seara, T.M.: The Melnikov method and subharmonic orbits in a piecewise smooth system. Siam. J. Appl. Dyn. Syst. 11(3), 801-830 (2012)

17. Granados, A., Hogan, S.J., Seara, T.M.: The scattering map in two coupled piecewise-smooth systems, with numerical application to rocking blocks. Phys. D. 269(7), 1-20 (2014)

18. Tian, R.L., Zhou, Y.F., Zhang, B.L.: Chaotic threshold for a class of impulsive differential system. Nonlinear Dyn. 83(4), 2229-2240 (2016)

19. Li, S.B., Gong, X.J., Zhang, W., Hao, Y.X.: The Melnikov Method for detecting chaotic dynamics in a planar hybrid piecewise-smoothsSystem with a switching manifold. Nonlinear Dyn. 89, 939-953 (2017)

20. Cao, Q.J., Wiercigroch, M., Pavlovskaia, E.E., Thompson, J.M.T., Grebogi, C.: Archetypal oscillator for smooth and discontinuous dynamics. Phys. Rev. E. 74(4), 046218 (2006) 
21. Cao, Q.J., Wiercigroch, M., Pavlovskaia, E.E., Thompson, J.M.T., Grebogi, C.: SD oscillator, the attractor and their applications. J. Vib. Eng. Technol. 20(5), 454-458 (2007)

22. Cao, Q.J., Wiercigroch, M., Pavlovskaia, E.E., Thompson, J.M.T., Grebogi, C.: Piecewise linear approach to an archetypal oscillator for smooth and discontinuous dynamics. Philos. Trans. R. Soc A. 366(1865), 635-652 (2008)

23. Cao, Q.J., Wiercigroch, M., Pavlovskaia, E.E., Thompson, J.M.T.: The limit case response of the archetypal oscillator for smooth and discontinuous dynamics. Int. J. Nonlinear Mech. 43, 462-473 (2008)

24. Shen, J., Li, Y.R., Du, Z.D.: Subharmonic and grazing bifurcations for a simple bilinear oscillator. Int. J. Nonlinear Mech. 60, 70-82 (2014)

25. Yi, T.T., Du, Z.D.: Degenerate grazing bifurcations in a simple bilinear oscillator. Int. J. Bifurcat. Chaos 24(11), 1450141 (2014) 